ARTICLE

https://doi.org/10.1038/s41467-019-11328-0

\title{
Reconfigurable two-dimensional optoelectronic devices enabled by local ferroelectric polarization
}

\author{
Liang Lv ${ }^{1}$, Fuwei Zhuge (1) ${ }^{1}$, Fengjun Xie ${ }^{1}$, Xujing Xiong ${ }^{1}$, Qingfu Zhang ${ }^{1}$, Nan Zhang ${ }^{1}$, Yu Huang ${ }^{1}$ \&
}

Tianyou Zhai (i) ${ }^{1}$

Ferroelectric engineered pn doping in two-dimensional (2D) semiconductors hold essential promise in realizing customized functional devices in a reconfigurable manner. Here, we report the successful pn doping in molybdenum disulfide $\left(\mathrm{MoS}_{2}\right)$ optoelectronic device by local patterned ferroelectric polarization, and its configuration into lateral diode and npn bipolar phototransistors for photodetection from such a versatile playground. The lateral pn diode formed in this way manifests efficient self-powered detection by separating 12\% photo-generated electrons and holes. When polarized as bipolar phototransistor, the device is customized with a gain $\sim 1000$ by its transistor action, reaching the responsivity $\sim 12 \mathrm{~A} \mathrm{~W}^{-1}$ and detectivity over $10^{13}$ Jones while keeping a fast response speed within $20 \mu \mathrm{s}$. A promising pathway toward high performance optoelectronics is thus opened up based on local ferroelectric polarization coupled 2D semiconductors.

\footnotetext{
${ }^{1}$ State Key Laboratory of Materials Processing and Die \& Mould Technology, School of Materials Science and Engineering, Huazhong University of Science and Technology, 430074 Wuhan, People's Republic of China. Correspondence and requests for materials should be addressed to F.Z. (email: zhugefw@hust. edu.cn) or to T.Z. (email: zhaity@hust.edu.cn)
} 
T wo-dimensional (2D) semiconductors have drawn extensive interests in functional electronic devices for their exotic optoelectronic properties by the quantum confinement in atomic thickness and feasibly changed characteristics under external modulation ${ }^{1-5}$. Using field effects coupled by high- $\kappa$ dielectrics ${ }^{6,7}$, ionic liquids and gels ${ }^{8,9}$, and ferroelectric (FE) polarization ${ }^{10-12}$, their electric properties have been widely tuned from semiconductor to highly conductive metals and oppositely to the insulators. Such features have enriched the emerging of various gate-modulated devices,13, including transistors ${ }^{14,15}$, logic inverters 7 , memories ${ }^{11,16}$, light-emitting diodes (LEDs) ${ }^{17}$, and photodetectors 3 ,18,19. An ultimate pursuit to this end would be however a reconfigurable functional device that can be customized on demand, so that a universal device architecture can be deployed in various applications. FE materialcoupled 2D semiconductors hold special promise in reforming these devices toward a reconfiguration, given their large, nonvolatile yet rewritable feature of remnant polarization ${ }^{20-23}$. There is an increasing interest in integrating $\mathrm{FE}$ materials, such as $\mathrm{BiFeO}_{3}{ }^{10}, \mathrm{P}(\mathrm{VDF}-\mathrm{TrFE})^{12}, \mathrm{PbZrTiO}_{3}{ }^{16,24}$, and $\mathrm{LiNbO}_{3}{ }^{25}$, to $2 \mathrm{D}$ functional devices. However, further efforts are, to a marked degree, hampered by the ability of FE polarization in controlling pn doping of $2 \mathrm{D}$ materials ${ }^{23}$, which constitutes a fundamental building block for functional electronic devices ${ }^{26,27}$.

Given the selective pn doping of $2 \mathrm{D}$ materials, a variety of fundamental devices that enable the digital technology can be developed, for example, pn diodes and amplifying bipolar transistors ${ }^{28,29}$. In optoelectronics, these devices based on pn junctions could manifest self-powered or fast and high gain photodetection beyond the usual photoconductors. However, early attempts to construct the kind of devices relied prominently on locally buried gates ${ }^{28}$, lateral and vertical heterojunctions $s^{30-33}$, the behavior of which are complicated to manipulate without fine-tuned pn doping. For example, the amplification gain in the resulting bipolar transistor is severely limited by the low carrier injection efficiency at the emitter-base junction, for example, based on $\mathrm{MoS}_{2} / \mathrm{WSe}_{2}{ }^{29}$ or $\mathrm{MoS}_{2} / \mathrm{BP}$ heterojunctions ${ }^{34}$. Moreover, these efforts fail to satisfy reconfigurable customization on demand with the predefined gate electrodes, epitaxial sequence in lateral junction, or stacking order in van der Waals heterostructures. With the large remnant polarization, FE coupling to $2 \mathrm{D}$ semiconductors is favored to realize reconfigurable optoelectronics based on rewritable pn doping in the $2 \mathrm{D}$ components, which however has not been demonstrated yet ${ }^{10,12,16}$. For example, organic FE materials, such as P(VDF-TrFE) copolymers, have in the past manifested indispensable merits in their facile spin-coating fabrication. Sophisticate top-gate integration methods have been adopted for their integration with various $2 \mathrm{D}$ semiconductors, including $\mathrm{MoS}_{2}{ }^{11,12,35,36}, \mathrm{MoSe}_{2}{ }^{37}, \mathrm{MoTe}_{2}$, and $\mathrm{WSe}_{2}{ }^{38}$. However, these efforts generally fail to achieve pn modulation even with the large remnant polarization of P(VDFTrFE) $\left(\sim 8 \mu \mathrm{C} \mathrm{cm}^{-2}\right)$ over conventional gate oxides, which draw increasing demand to improve the $\mathrm{FE}$ integration in $2 \mathrm{D}$ optoelectronic devices for reconfigurable pn doping and functionalization.

Here, we report on reconfigurable optoelectronic photodetectors with FE polarization-defined pn doping in $\mathrm{MoS}_{2}$ using the device configuration of bottom electrical contacts. With the rewritable FE polarization using a scanning atomic force microscope (AFM) tip, the $\mathrm{MoS}_{2}$ photoconductor is facile customized into pn diodes and bipolar phototransistors with optimal photodetection performance. This allows us to demonstrate a lateral pn diode with an ideal factor of 1.7 for self-powered photodetection that separate $\sim 12 \%$ photogenerated electrons and holes, and a npn bipolar phototransistor with a gain $\sim 1000$ with fine-adjusted pn polarization. Such reconfigurable device characteristics may promote the evolvement of smart image sensors that respond to external light levels for balanced photoresponse gain and energy efficiency. Our study on reconfigurable optoelectronic devices using FE polarization thus unravels a pathway towards customizing novel functional optoelectronics based on 2D semiconductors.

\section{Results}

Reconfigurable pn doping in $\mathrm{MoS}_{2}$ by FE polarization. Figure 1a illustrates the configuration of FE-coupled $\mathrm{MoS}_{2}$ device, in which an AFM tip is employed to switch the local polarization. To fabricate the device, multilayer $\mathrm{MoS}_{2}$ from mechanical exfoliation is used as the semiconductor channel, while the FE copolymer $\mathrm{P}(\mathrm{VD}-\mathrm{TrFE})$ is spin coated on the top with a thickness of $\sim 200 \mathrm{~nm}$. The copolymer film is later crystallized into its orthorhombic $\beta$ phase by annealing at $135^{\circ} \mathrm{C}$ for $15 \mathrm{~min}^{39}$. The coercive field of as-prepared $\mathrm{P}(\mathrm{VDF}-\mathrm{TrFE})$ film, under which the FE polarization switches, is measured in agreement with literature to be $\sim 5 \times 10^{7} \mathrm{~V} \mathrm{~m}^{-1}$ using capacitance-voltage measurement (Supplementary Fig. 1) $)^{40}$. The FE nature of $\mathrm{P}(\mathrm{VDF}-\mathrm{TrFE})$ in intimate contact with $\mathrm{MoS}_{2}$ is then exploited to engineer the carrier doping in $\mathrm{MoS}_{2}$ through their reversible polarization by an external poling field. The representative coupling between the FE polarization in $\mathrm{P}(\mathrm{VDF}-\mathrm{TrFE})$ and the adjacent $\mathrm{MoS}_{2}$ is illustrated in Fig. 1b. To obtain p-type doping in $\mathrm{MoS}_{2}$, upward polarization $(\mathbf{P} \uparrow)$ in $\mathrm{P}(\mathrm{VDF}-\mathrm{TrFE})$ is required, while reversely the opposite downward polarization $(\mathbf{P} \downarrow)$ is desired to enhance $\mathrm{n}$-type doping. Specifically, in order to achieve widely tuned doping in $\mathrm{MoS}_{2}$, we adopt bottom electrode contacts by placing thin $\mathrm{MoS}_{2}$ flakes on top of predefined metal electrodes, as indicated in Fig. 1a. An AFM image of the bare device 1 (Dev-1) with 2.4-nm-thick $\mathrm{MoS}_{2}$ transferred onto $\mathrm{Cr} / \mathrm{Au}$ electrodes is shown in Fig. 1c (before spin coating the $\mathrm{P}(\mathrm{VDF}-\mathrm{TrFE})$ layer). Table 1 lists the parameters of $\mathrm{MoS}_{2}$ devices studied in this work. Compared to the previous investigations with metal electrodes on the top of $\mathrm{MoS}_{2}$ or other 2D materials ${ }^{35,38,41}$, which may screen the FE polarization field near contact and result in Schottky barriers, this will ensure the intimate coupling of the FE polarization field to $\mathrm{MoS}_{2}$ at the contacts and hence uniform doping to the whole semiconductor channel.

To switch the FE polarization in $\mathrm{P}(\mathrm{VDF}-\mathrm{TrFE})$, an AFM system is employed for the facile reconfiguration of polarization pattern, as reported in the literature ${ }^{22}$. Despite the switched FE polarization tends to relax due to the incomplete compensation to depolarization field in $\mathrm{P}(\mathrm{VDF}-\mathrm{TrFE})$, it enables rewritable polarization pattern on the same device, thereby allowing the direct study of the influence of device configurations without worrying about material differences. To pole the FE layer, the AFM tip is grounded while the source (S) and drain (D) electrodes of $\mathrm{MoS}_{2}$ channel are biased by a poling voltage $V_{\mathrm{p}}$. By scanning the AFM tip over the device area, the P(VDF-TrFE) copolymer between the tip and $\mathrm{MoS}_{2}$ channel is polarized to $\mathbf{P} \uparrow$ using positive $V_{\mathrm{p}}$ and $\mathbf{P} \downarrow$ with negative $V_{\mathrm{p}}$. The effect of resulted remnant $\mathrm{FE}$ polarization in $\mathrm{P}(\mathrm{VDF}-\mathrm{TrFE})$ to the electrical conductance of $\mathrm{MoS}_{2}$ in Dev-1 is reflected by the significant hysteresis shown in Fig. 1d, which is measured with $V_{\mathrm{ds}}=1 \mathrm{~V}$ after each polarization scan. With positive $V_{\mathrm{p}}$, the current flow in $\mathrm{MoS}_{2}$ is dramatically decreased with increasing $V_{\mathrm{p}}$ due to the depletion of electrons. However, an apparent change is observed with $V_{\mathrm{p}}>+20 \mathrm{~V}$, that is, increasing the poling voltage leads to increased conductance. Such transition is consistent with a reversal of the doping polarity in $\mathrm{MoS}_{2}$ to $\mathrm{p}$-type due to the dramatically increased remnant polarization $(\mathbf{P} \uparrow)$ in $\mathrm{FE}$ film ${ }^{16}$. This is confirmed by the field-effect modulation measurements using the Si back-gate, as will be discussed later. By wweeping the 
a

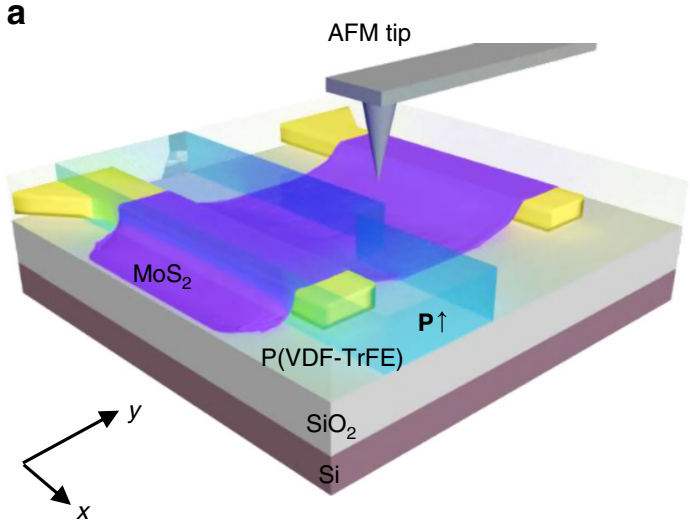

C

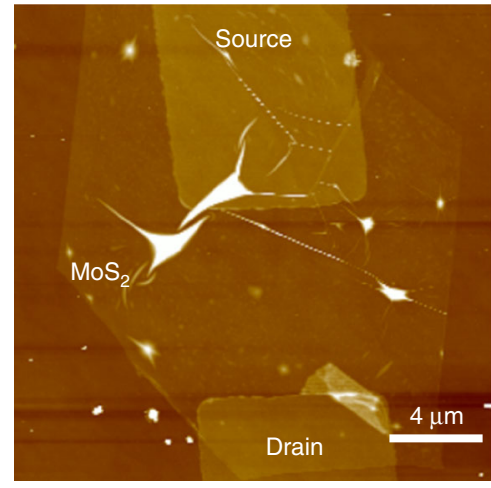

b

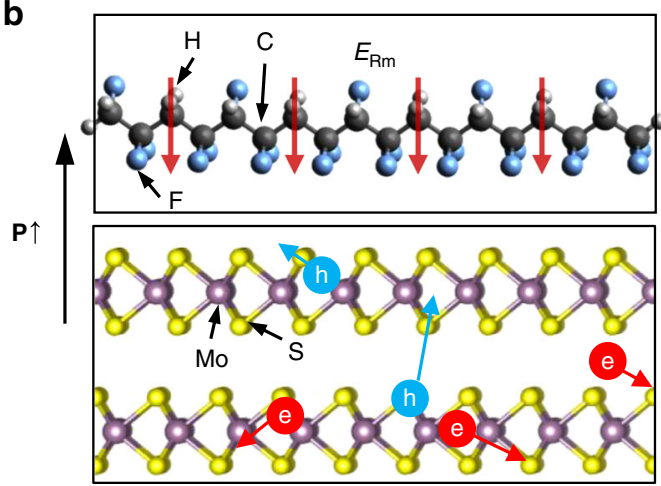

d

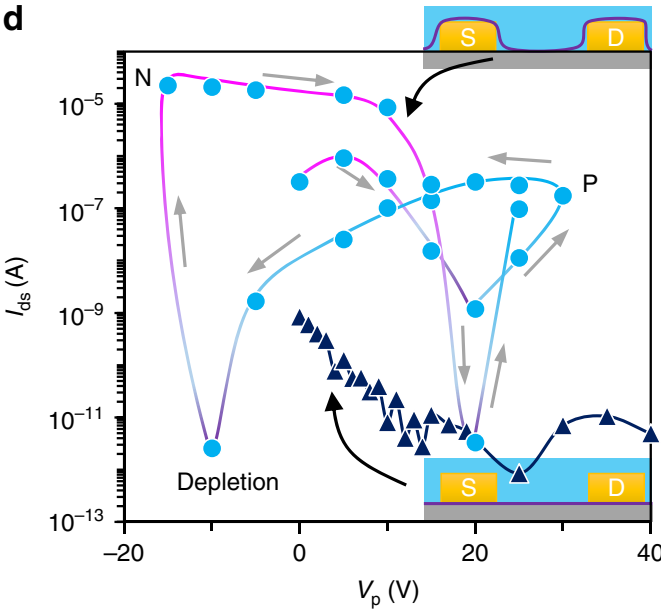

Fig. 1 Ferroelectric polarization enabled $p$ and $n$ doping switch of $\mathrm{MoS}_{2}$. a Configuration of ferroelectric copolymer-coupled MoS 2 device using a scanning atomic force microscope (AFM) tip as the poling electrode, the blue colored area becomes upward polarized (P $\uparrow$ ) after applying positive bias on device while keeping the scanning AFM tip as ground. $\mathbf{b}$ The coupling between $\mathbf{P} \uparrow$ polarization in ferroelectric copolymer and carriers in MoS $_{2}$ resulting in accumulated holes near the interface. c AFM image of a $\mathrm{MoS}_{2}$ thin flake in device 1 (Dev-1) transferred onto predefined source and drain electrodes made by $10 \mathrm{~nm} \mathrm{Cr} / \mathrm{Au}$. d Hysteresis variation of the $\mathrm{MoS}_{2}$ conductance when sweeping the poling voltages $\left(V_{\mathrm{p}}\right)$ under different device configurations: with the electrode contacts defined at the bottom (circle) and on the top (triangle) of $\mathrm{MoS}_{2}$, respectively

Table 1 Parameters of $\mathrm{MoS}_{2}$ devices and their defined functions in this work

\begin{tabular}{lllll} 
Device & Length $(\boldsymbol{\mu} \mathbf{m})$ & Width $\mathbf{(} \boldsymbol{\mu} \mathbf{m})$ & Thickness $(\mathbf{n m})$ & Defined functions \\
\hline Dev-1 & 8.0 & 7.8 & 2.4 & p, n, pn diode \\
Dev-2 & 7.6 & 3.2 & 3.8 & pn diode, npn transistor \\
Dev-3 & 6.9 & 4.7 & 3.4 & pn diode, npn transistor \\
Dev-4 & 7.5 & 3.0 & 4.4 & npn transistor \\
\hline
\end{tabular}

poling voltage to negative, an opposite transition from $\mathrm{p}$ to $\mathrm{n}$ happens at $V_{\mathrm{p}}<-10 \mathrm{~V}$. These turning voltages are found consistent with the previous measured coercive voltages of $\mathrm{P}$ (VDF-TrFE) films, thus validating the correlation between the changed pn doping states in $\mathrm{MoS}_{2}$ and the FE polarization switching. It should be noted that the successful doping polarity switching in $\mathrm{MoS}_{2}$ is in sharp contrast with the one that employed electrode-on-top configuration, which in our control experiment displays only monotonous decrease of conductance with increasing $V_{\mathrm{p}}$ (see Fig. 1d). This confirms the vital role of electrode contact configuration in ensuring the doping polarity engineering in $\mathrm{MoS}_{2}$ and agrees well with the early conclusion that the doping in $2 \mathrm{D}$ semiconductors may be retarded by the Schottky barrier near metal contacts ${ }^{42,43}$. The universality of the above-adopted strategy is further demonstrated by its application in few-layer $\mathrm{WSe}_{2}(4.2 \mathrm{~nm})$ (Supplementary Fig. 2), which manifests nearly symmetric $\mathrm{p} / \mathrm{n}$ doping transition at $V_{\mathrm{p}}= \pm 6 \mathrm{~V}$ because of its bipolar characteristic.

With the present re-designed electrode configuration, the conductance of $\mathrm{MoS}_{2}$ channel can be modulated with an on-off ratio of $>10^{7}$ as shown in Fig. 1d. Their corresponding current-voltage $(I-V)$ characteristics can be found in Supplementary Fig. 3. This modulation ratio is notably 2-3 orders higher than any of the previous reports because the significantly enhanced $\mathrm{ON}$ current in the $\mathrm{MoS}_{2}$ channels by avoiding the contact issue $^{12}$. To get further insight into the resulting doping characteristics in $\mathrm{MoS}_{2}$ by the coupled FE polarization, we analyze the field-effect measurements using the Si back-gate. Figure 2a displays the transfer curves of the $\mathrm{MoS}_{2}$ back-gate transistor after poling operations at $V_{\mathrm{p}}=0,-15$, and $+25 \mathrm{~V}$. The initial $\mathrm{MoS}_{2}$ is seen $\mathrm{n}$ doped, that is, increasing $V_{\mathrm{g}}$ at back-gate enhanced its conductance, while after $-15 \mathrm{~V}$ downward 

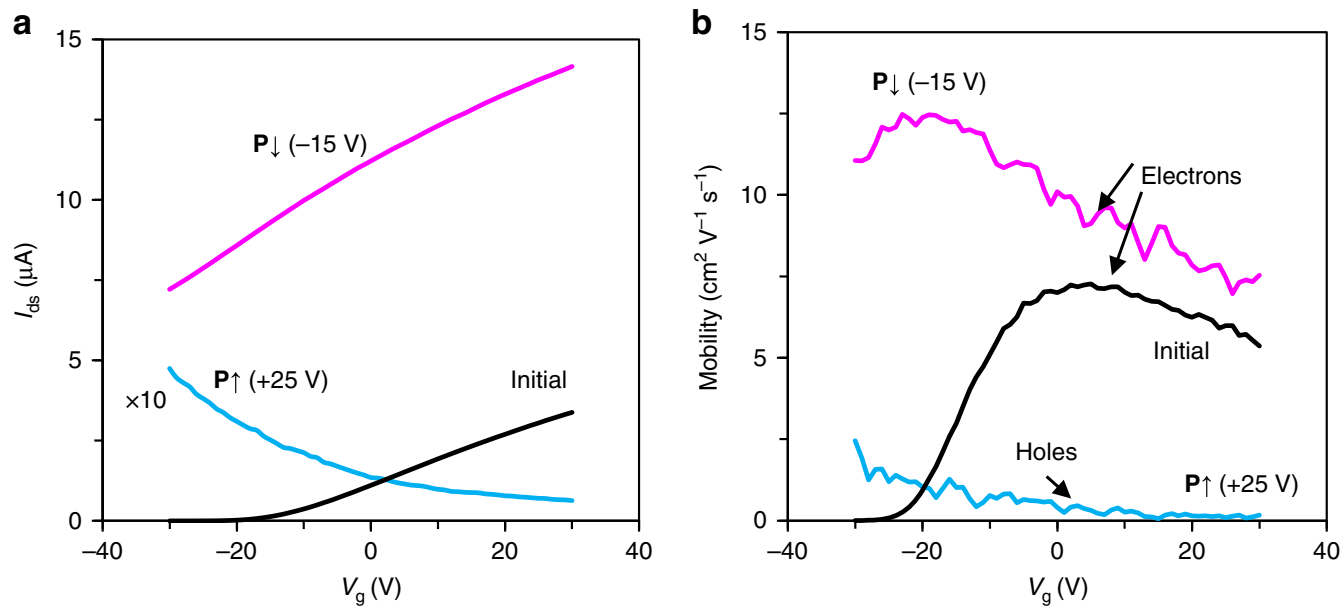

Fig. 2 Field-effect characteristic of $p$ - and n-doped $M_{0} S_{2}$ under different ferroelectric polarization states. a Transfer curves of the MoS ${ }_{2}$ device 1 (Dev-1) modulated by Si back-gate measured at a constant source-drain bias $V_{\mathrm{ds}}=1 \mathrm{~V}$. The device is measured in several polarization states including the initial state and after upward $\left(\mathbf{P} \uparrow\right.$, by a poling voltage $\left.V_{p}=+25 \mathrm{~V}\right)$ and downward $\left(\mathbf{P} \downarrow\right.$, by $\left.V_{p}=-15 \mathrm{~V}\right)$ ferroelectric polarization. $\mathbf{b}$ The corresponding mobility for electrons and holes extracted from field-effect measurements. The decrease of mobility when increasing gate bias for electrons is related to the electron-electron scattering limited mobility in degenerately n-doped $\mathrm{MoS}_{2}$

polarization $(\mathbf{P} \downarrow)$, its threshold voltage is remarkably shifted to negative from -13 to $-85 \mathrm{~V}$. Considering the oxide capacitance of $300 \mathrm{~nm} \mathrm{SiO}, C_{\mathrm{ox}}=11.5 \mathrm{nF} \mathrm{cm}^{-2}$, the FE-coupled effective charge carriers in $\mathrm{MoS}_{2}$ is estimated to be $\sim 0.8 \mu \mathrm{C} \mathrm{cm}^{-2}$ by $\Delta Q=$ $\Delta V_{\mathrm{th}} \cdot C_{\mathrm{ox}}{ }^{44}$, which accounts to only a portion $(\sim 10 \%)$ of the remnant polarization of $\mathrm{P}(\mathrm{VDF}-\mathrm{TrFE})$. This indicates that a majority of coupled electrons were frozen in new deep energy traps formed after polarization, for example, the acceptor states by the prompt interaction between aligned F-containing groups and $\mathrm{MoS}_{2}{ }^{45}$. However, the mobility in $\mathrm{MoS}_{2}$ is not influenced as the traps are effectively screened by the heavy concentration of electron carriers. Figure $2 \mathrm{~b}$ displays the extracted carrier mobility in $\mathrm{MoS}_{2}$ with respect to different FE polarization, calculated using $\mu=(L / W) V_{\mathrm{ds}}{ }^{-1} C_{\mathrm{ox}}{ }^{-1}\left(\mathrm{~d} I_{\mathrm{ds}} / \mathrm{d} V_{\mathrm{g}}\right)$, where $L$ and $W$ are, respectively, the channel length and width. The maximum electron mobility in $\mathrm{MoS}_{2}$ is $\sim 12.3 \mathrm{~cm}^{2} \mathrm{~V}^{-1} \mathrm{~s}^{-1}$ under $\mathbf{P} \downarrow$. Notably, increasing $V_{\mathrm{g}}$ in positive leads to the decrease of electron mobility in $\mathrm{n}$-doped $\mathrm{MoS}_{2}$ due to the dominant electron-electron scattering, implying that the FE polarization-enhanced n-type doping in $\mathrm{MoS}_{2}$ is reaching a heavily doped metallic behavior ${ }^{6}$. In comparison, the $\mathrm{MoS}_{2}$ after $25 \mathrm{~V}(\mathbf{P} \uparrow)$ poling manifests apparent p-type behavior, with the hole mobility decreasing with increasing $V_{\mathrm{g}}$. Such transport behavior is attributed to the trap-dominated transport in p-type $\mathrm{MoS}_{2}$, since the inherent donor atoms in $\mathrm{MoS}_{2}$ start to act as deep energy traps to holes ${ }^{46}$. By using the carrier mobility extracted from transfer curves at each $\mathrm{FE}$ polarization state, the free carrier concentration tuned by FE polarization is estimated to be $\sim 10^{9}-10^{12} \mathrm{~cm}^{-2}$ in $\mathrm{MoS}_{2}$ for both electrons and holes, and in $\mathrm{WSe}_{2} \sim 10^{7}-10^{11} \mathrm{~cm}^{-2}$ (Supplementary Note 1, Supplementary Fig. 4, and Supplementary Table 1). The reversibly and significantly tuned $\mathrm{p} / \mathrm{n}$ doping and large ON/ OFF switch ratio covering metallic, semiconductor, and insulator behaviors will promote their potential applications in various optoelectronic devices with reprogrammable functions.

Pn diode by locally defined FE polarization pattern. Based on the FE polarization-enabled $\mathrm{p}$ and $\mathrm{n}$ doping in $\mathrm{MoS}_{2}$, we construct a lateral pn junction using locally patterned polarization as illustrated in Fig. 3a. The bias voltage on the $\mathrm{MoS}_{2}$ channel in experiment is programmed to switch from +25 to $-15 \mathrm{~V}$, while the tip scans between the $\mathrm{S}$ and $\mathrm{D}$ electrode, resulting in a polarization pattern shown in Fig. $3 \mathrm{~b}$, depicted by the phase image in piezoelectric force microscope (PFM). Note that the expected transition region of remnant polarization ( $\mathbf{P} \uparrow$ to $\mathbf{P} \downarrow$ ) by switching the polarity of bias voltage is estimated to be $\sim 100-180$ nm (Supplementary Fig. 5, limited by the electric field distribution near AFM tip and the FE domain size in P(VDF-TrFE) at a thickness of $200 \mathrm{~nm})$. Figure $3 \mathrm{c}$ displays the $I-V$ characteristic of $\mathrm{MoS}_{2}$ channel in Dev-2 with three kinds of FE field-effect doping configurations, that is, complete $\mathrm{p}$ and $\mathrm{n}$ doping on the device area, and patterned lateral pn junction. For both the n- and pdoped device, the electrical contact shows Ohmic behavior due to the degenerate doping in $\mathrm{MoS}_{2}$. However, we note that for moderately p-doped $\mathrm{MoS}_{2}$, nonlinear $I-V$ characteristics may appear with ohmic contact at low bias conditions, but space charge-limited current (SCLC) at large biases, as revealed in Supplementary Fig. 3. Such behavior is however attributed to the charge trapping in channel upon intense hole injection under large external bias ${ }^{47}$. Nevertheless, SCLC behavior is alleviated in heavily p-doped devices by the filling of trap centers when the Fermi energy $E_{\mathrm{F}}$ is close to the valance band maximum. With laterally patterned $\mathrm{p}$ and $\mathrm{n}$ doping of the channel, the formed $\mathrm{pn}$ diode exhibits clear rectification behavior with a large on-off ratio of $\sim 10^{5}$ at $\pm 1 \mathrm{~V}$. The value is higher than the $\mathrm{MoS}_{2} / \mathrm{GaTe}$ heterojunction ${ }^{48}$ and larger than the lateral $\mathrm{MoS}_{2}$ homojunction enabled by chemical doping ${ }^{49}$, which are attributed to the widely tuned doping concentration in $\mathrm{MoS}_{2}$ together with the electrical contact. By fitting the junction current using

$$
I=I_{0} e^{e\left(V-I R_{\mathrm{s}}\right) / n k_{\mathrm{B}} T},
$$

where $I_{0}, R_{\mathrm{s}}$, and $n$ denotes, respectively, the reverse saturation current, series resistance, and ideal factor. The formed pn junction is found to exhibit an ideal factor of 1.7 and $R_{\mathrm{s}} \approx 10 \mathrm{M} \Omega$. With $n \sim 2$, the junction current shall be dominated by electron-hole recombination in the space charge region ${ }^{50}$. The recombination could however be optimized by, for example, improving the collection efficiency of metal electrodes by minimizing the size of the $\mathrm{p}$ region. In our work, the ideal factor is reduced to 1.4 when reducing the p-doped region in junction (Supplementary Note 2, Supplementary Fig. 6-8), which implies that within the FEdefined pn diode the majority of the charge recombination occurred at the p-doped region.

When illuminating the pn diode with $532 \mathrm{~nm}$ light, apparent self-driven photocurrent is observed under zero bias driven by the 


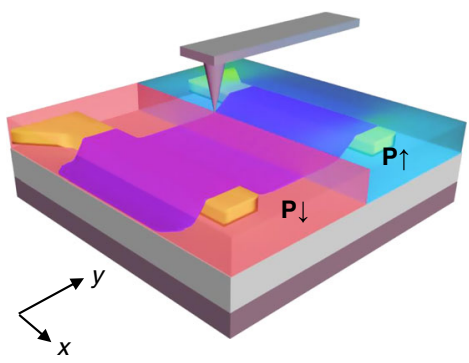

d

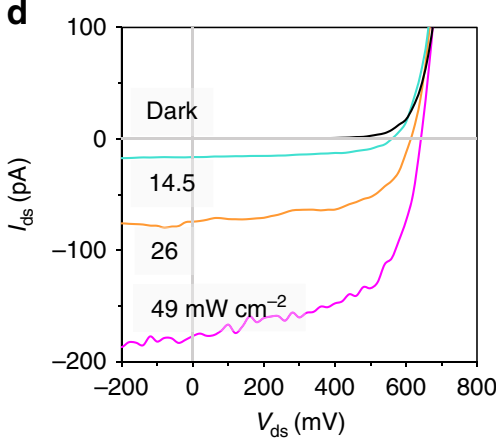

b
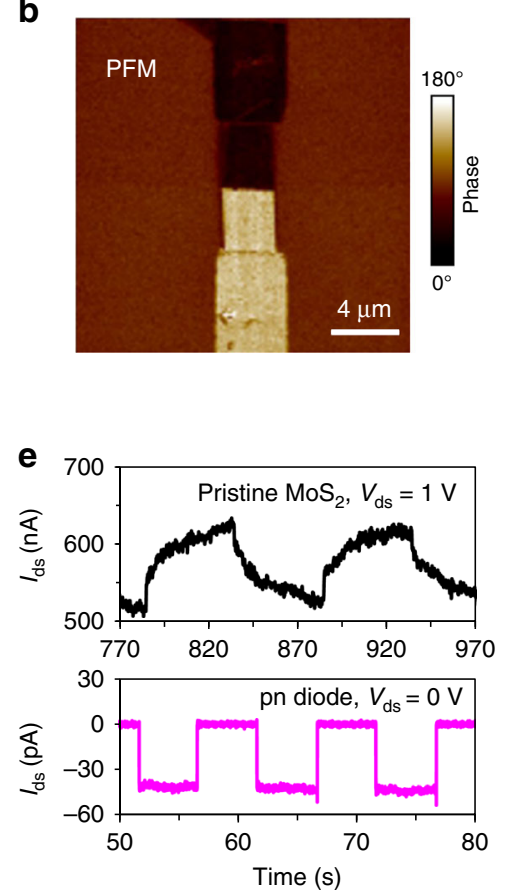

c

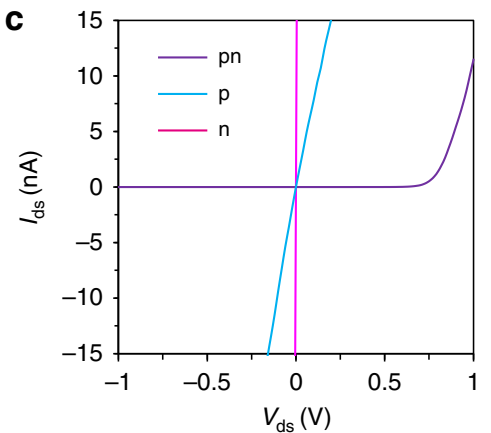

f
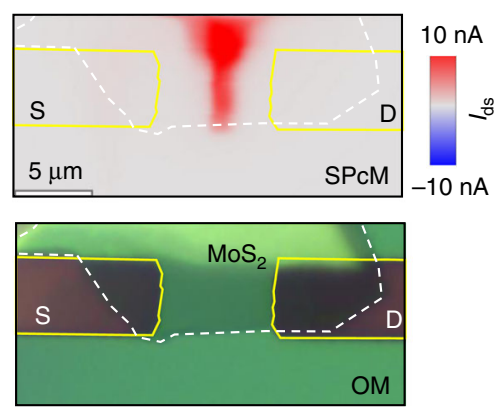

Fig. $3 \mathrm{MoS}_{2}$ pn junction defined by locally patterned ferroelectric polarization and its characteristics. a Formation principle of $\mathrm{MoS}_{2}$ pn junction by locally patterned upward $(\mathbf{P} \uparrow)$ and downward $(\mathbf{P} \downarrow)$ ferroelectric polarization using a scanning AFM tip. $\mathbf{b}$ Piezoelectric force microscope (PFM) phase image of the formed FE polarization pattern on device 2 (Dev-2). The bright and dark-colored region correspond, respectively, to the polarization states of $\mathbf{P} \uparrow$ and $\mathbf{P} \downarrow$ in FE copolymer, which cause, respectively, $\mathrm{p}$ - and $\mathrm{n}$-type doping to $\mathrm{MoS}_{2} \cdot \mathbf{c} I-V$ characteristic of the pn-doped $\mathrm{MoS}_{2}$ channel compared to complete $\mathrm{p}$ and $\mathrm{n}$ doping. d $I-V$ characteristic of the $M_{0}$ pn junction under $532 \mathrm{~nm}$ laser illumination of different intensities, from dark to $14.5,26$, and $49 \mathrm{~mW} \mathrm{~cm}^{-2}$, showing photovoltaic-like behavior. e Transient photoresponse of the self-powered pn junction compared to the pristine MoS $_{2}$ device in photoconductor configuration. $\mathbf{f}$ Spatial photocurrent map of Dev-3 in short circuit compared to its optical microscope image. Self-driven photocurrent is confirmed to generate from the pn junction defined in the middle of channel rather than from contact effects

built-in field in the junction as can be seen in Fig. 3d. In comparison, devices with complete $\mathrm{n}$ or $\mathrm{p}$ doping across the channel do not display such self-driven photocurrent under illumination (Supplementary Fig. 9). The open-circuit voltage $\left(V_{\mathrm{oc}}\right)$ of the FE polarization-defined pn junction is $\sim 650 \mathrm{mV}$ with light intensity $>10 \mathrm{~mW} \mathrm{~cm}^{-2}$, which is comparable to silicon diodes and approximately half of the indirect bandgap $(1.2 \mathrm{eV})$ of few-layer $\mathrm{MoS}_{2}$. The large $V_{\mathrm{oc}}$ suggests efficient charge separation in devices enabled by the intense lateral built-in electric field across the pn diode. In the short-circuit condition, the photocurrent is $\sim 177 \mathrm{pA}$ at an intensity of $49 \mathrm{~mW} \mathrm{~cm}^{-2}$, indicating a self-powered responsivity $R \approx 15 \mathrm{~mA} \mathrm{~W}^{-1}(R=\alpha \eta$ $(e / h v)$, where $\alpha$ and $\eta$ are, respectively, the light absorption efficiency and internal quantum efficiency in device). Considering that $3.8 \mathrm{~nm} \mathrm{MoS}_{2}$ absorbs $\sim 30 \%$ of the incident photons at 532 $\mathrm{nm}^{51}, \eta$ is estimated to be $12 \%$ in the diode, which could be optimized by defining the junction close to electric contact, so as to minimize the serial resistance in the channel. Given the large $V_{\mathrm{oc}}$ and a fill factor of $\sim 0.58$, an overall power conversation efficiency of $\sim 0.61 \%$ is achieved. We note that the value outperforms that of vertically stacked $\mathrm{GaTe} / \mathrm{MoS}_{2}$ heterojunction ${ }^{48}, \mathrm{MoS}_{2}$ pn junctions ${ }^{52}$, and the lateral $\mathrm{WSe}_{2} / \mathrm{MoS}_{2}$ pn heterojunctions ${ }^{53}$, suggesting the attractive potential of FEcoupled pn junctions. As indicated in Fig. 3e, the efficient photovoltaic separation of electrons and holes renders rapid selfpowered photoresponse in photodetection over conventional photoconductor devices. The self-powered $\mathrm{MoS}_{2}$ device exhibits fast response within 10-20 $\mu$ s (Supplementary Fig. 10), being $~ 6$ orders of magnitude faster than the pristine $\mathrm{MoS}_{2}$ that suffered persistent photoconductance by the long-lasting photogate effects from trap states ${ }^{54}$.
To elucidate the self-powered photocurrent generation, spatially resolved photocurrent distribution has been measured for a short-circuited pn diode defined in Dev-3. This is achieved by locally illuminating the device using a fine laser spot $(\lambda=532$ $\mathrm{nm}$, focused spot diameter $\approx 500 \mathrm{~nm}$ ) in a confocal microscope. The pn junction is defined in the middle of $\mathrm{MoS}_{2}$ channel. Figure $3 \mathrm{f}$ displays the optical microscope image of the device and the associated photocurrent map. It is clear that most of the photocurrent is generated near the defined junction. We note that the self-driven photocurrent may also appear in Schottky-contacted devices, but usually with reversed polarity near the source and drain electrodes due to opposite charge separation ${ }^{55-57}$. However, in all the devices we have studied, photocurrent barely appears near the contact electrodes and there is no change on the photocurrent polarity across the device area. These results thus validates the role of FE polarization-defined pn diode in bringing the high rectification in $I-V$ characteristics and self-driven photocurrent.

High gain npn bipolar transistor by FE polarization. Although pn diodes exhibit remarkably improved photoresponse speed over photoconductive devices, their responsivity is limited due to the loss of gain mechanism ${ }^{58}$. Here, with pn junction as the fundamental building block, we further construct a bipolar phototransistor in which the fast photovoltaic photocurrent can be amplified via its transistor action ${ }^{34}$. Compared to the avalanche photodetectors (APDs), the bipolar phototransistor could work at considerably lower operation voltages $(\sim 150 \mathrm{~V}$ for commercial Si APDs) while yielding the similar photodetection gain. A typical bipolar transistor consists of emitter (E), base (B), and collector (C) regions that are, respectively, $n, p$, and $n$ doped by $F E$ 
a

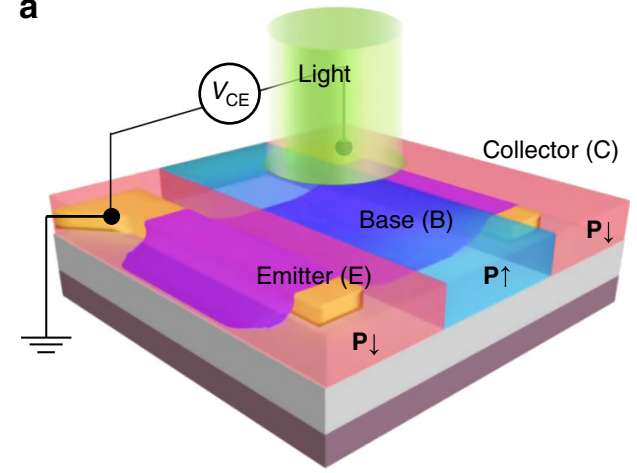

C

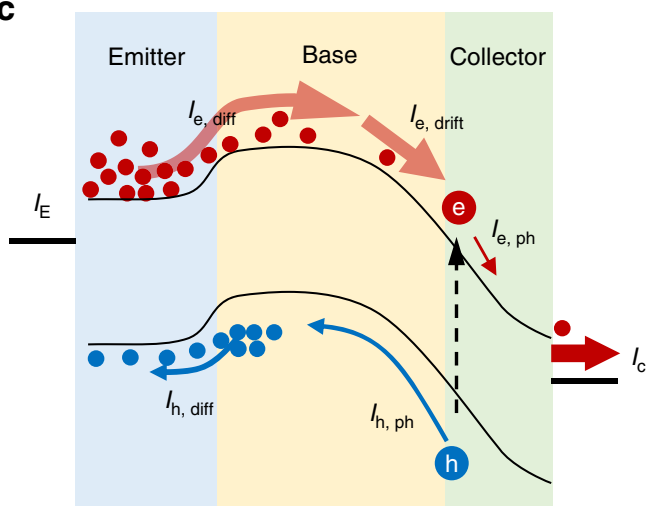

b

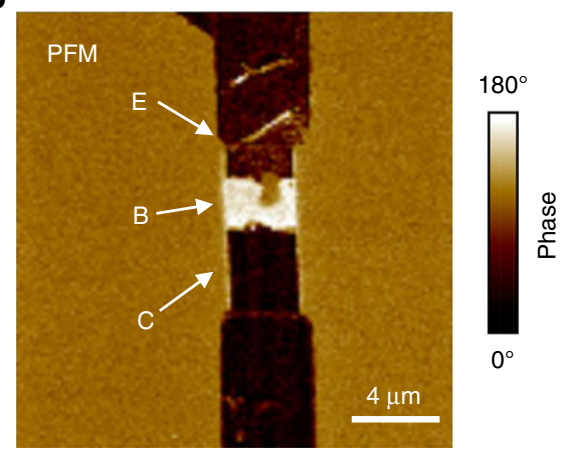

d

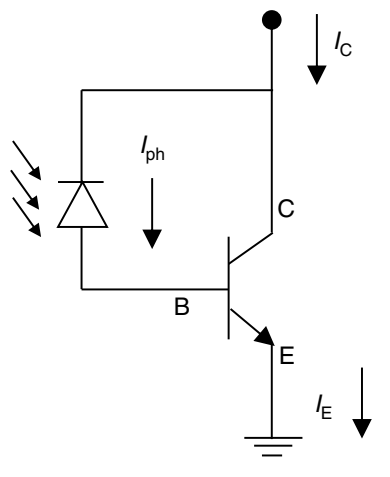

Fig. $4 \mathrm{MoS}_{2}$ bipolar phototransistor defined by ferroelectric polarization. a Schematic illustration of the fabrication of npn phototransistor by laterally patterned ferroelectric polarization ( $\mathbf{P} \downarrow, \mathbf{P} \uparrow$, and $\mathbf{P} \downarrow$ ) across the device, forming, respectively, the emitter (E), base (B), and collector (C) of the bipolar transistor. $\mathbf{b}$ Piezoelectric force microscope (PFM) phase image of the ferroelectric polarization pattern in device 2 (Dev-2) to make a MoS 2 bipolar transistor. c The energy band diagram and $\mathbf{d}$ equivalent circuit illustration of the working principle of a npn phototransistor under bias. The photovoltaic current $\left(I_{\mathrm{ph}}=I_{\mathrm{e}, \mathrm{ph}}+I_{\mathrm{h}, \mathrm{ph}}\right)$ at the reverse biased base-collector $(\mathrm{B}-\mathrm{C})$ junction is amplified by the large electron injection flux ( $I_{\mathrm{e}}$, diff $)$ at the forward biased emitter to base junction $(E-B)$, which is swept to collector when reaching the reverse biased $B-C$ junction. The collector current $I_{C}$ is finally amplified from photovoltaic current $I_{\text {ph }}$ by a factor of $\beta \approx I_{\mathrm{e}, \text { diff }} / I_{\mathrm{h} \text {,diff }}$ related to the electron and hole doping concentration in the emitter and base

polarization, as illustrated in Fig. 4a. The PFM phase image of the polarization pattern defined on Dev-2 for a npn bipolar transistor is displayed in Fig. 4b. For photodetection, the as-formed bipolar transistor is operated in a common-emitter mode with the base floated and collector positively biased. Since the transistor has the structure of two reversely connected pn junctions, it exhibits low dark current similar to that of a reversely biased photodiode. However, substantial gain could be obtained in the bipolar transistor to the photovoltaic current at the reverse biased $\mathrm{B}-\mathrm{C}$ junction ${ }^{28}$. This is elucidated by the energy band diagram in Fig. $4 \mathrm{c}$, where E-B junction is forward biased and $\mathrm{B}-\mathrm{C}$ junction is reverse biased under the positive collector bias. Upon illumination, the photogenerated electrons and holes within the $\mathrm{B}-\mathrm{C}$ junction are separated with electrons drifting to the n-type collector while holes drift to the p-type base, thus giving rise to the photocurrent $I_{\mathrm{ph}}$ as that in the photodiode. The holes separated into the base then diffuse across the forward biased $\mathrm{E}-\mathrm{B}$ junction

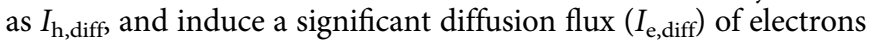
in the opposite direction. Once the electrons diffuse across the base and reach the reverse biased B-C junction, they will be swept and collected to the collector terminal by the built-in electric field, therefore contributing to the overall collector current. The collector current $I_{\mathrm{C}}$ is then amplified from the initial photocurrent $I_{\mathrm{ph}}$ by the injected electrons from $\mathrm{E}$. With $I_{\mathrm{C}}=(1+\beta) \cdot I_{\mathrm{ph}}, \beta$ is known as the gain factor that qualifies the performance of bipolar transistor, which is generally determined by the electron injection efficiency over the reverse hole current at the forward biased E-B

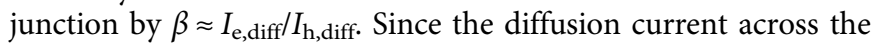

forward biased junction is governed by the majority carrier density over the space charge region, $\beta$ is intimately related to the doping concentration in the $\mathrm{E}$ and $\mathrm{B}$ with $\beta \propto N_{\mathrm{e}} / P_{\mathrm{b}}$, where $N_{\mathrm{e}}$ and $P_{\mathrm{b}}$ are, respectively, the electron and hole density in $\mathrm{E}$ and $\mathrm{B}^{59}$. The operation principle of such bipolar phototransistor is illustrated by the equivalent circuit shown in Fig. $4 \mathrm{~d}$, in which the diode corresponds to the reversely biased $\mathrm{B}-\mathrm{C}$ junction and the transistor represents the amplification of photodiode current by the forward biased E-B junction. In the present case, due to doping compensation effect in $\mathrm{MoS}_{2}$ by the inherent donor atoms, hole doping by FE coupling is naturally less significant than the case of electrons. A substantial gain factor to the photocurrent could be in principle obtained by constructing a npn transistor based on FE polarization.

Figure $5 \mathrm{a}$ shows the photoresponse behavior of the as-formed npn bipolar transistor to $532 \mathrm{~nm}$ laser illumination with varied intensity ranging from 0.02 to $39 \mathrm{~mW} \mathrm{~cm}^{-2}$. Once configured into bipolar transistor, the photocurrent is found remarkably improved in comparison to the previous photodiode along with a fast response speed. Such improvement is consistent with the expected behavior of bipolar transistor that amplifies photocurrent with high gain. By using a fast-switching 365-nm LED source, the photoresponse speed of the bipolar transistor is estimated to be $\sim 20 \mu$ s (see inset of Fig. 5a), making it one of the fastest $\mathrm{MoS}_{2}$ photodetectors, but with high gain characteristics. Faster response within as short as $\sim 3-5 \mu$ s is also achieved in experiments (Supplementary Fig. 11), which is close to the switching limit of the adopted light source. It is believed that the 
a

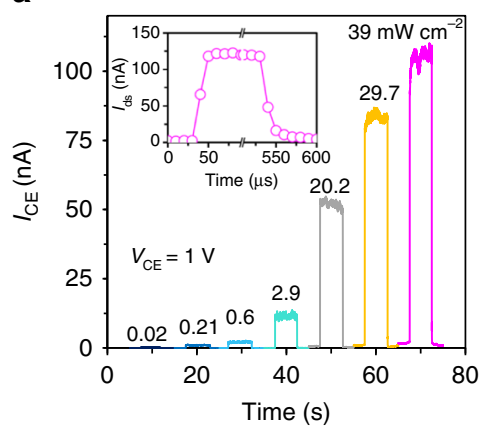

d
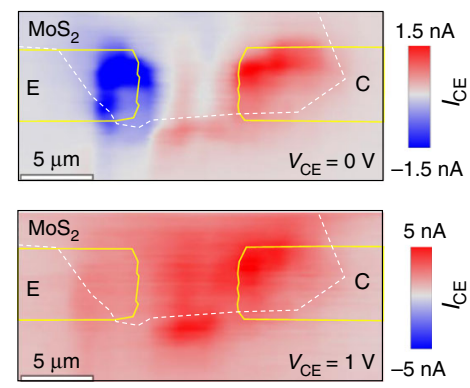

b

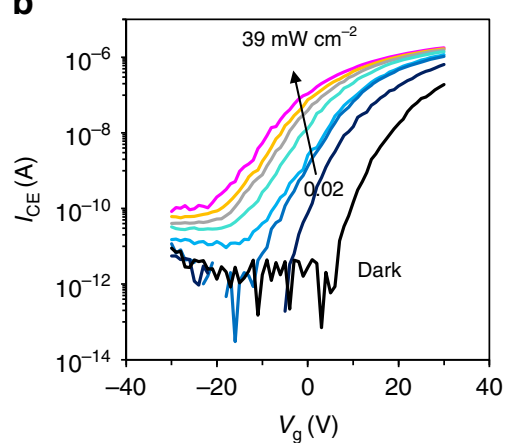

e

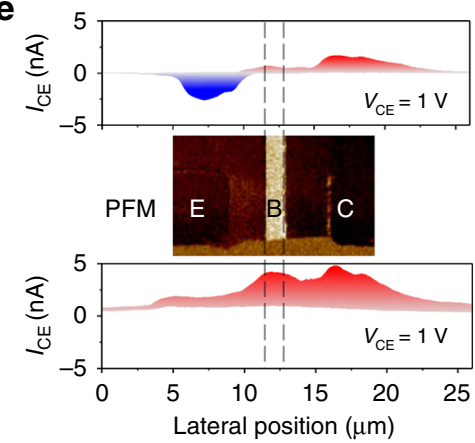

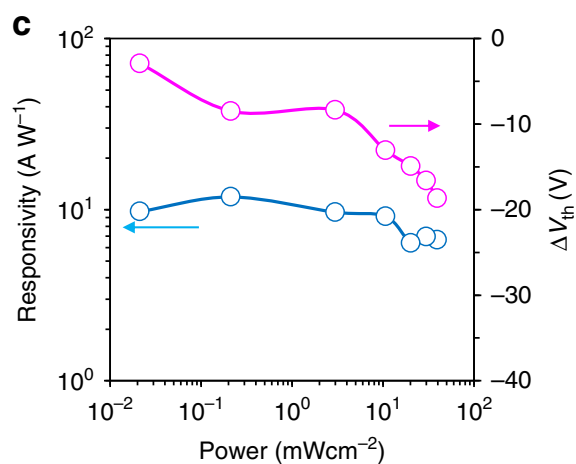

f

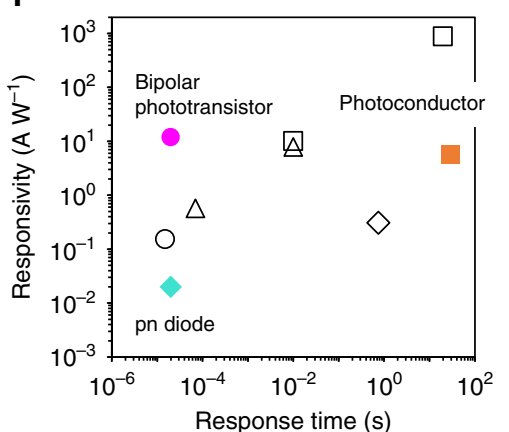

Fig. 5 Photodetection performance of $\mathrm{MoS}_{2}$ bipolar phototransistor. a Photoresponse of ferroelectric polarization-coupled MoS 2 npn transistor (device 2 $(\mathrm{Dev}-2)$ ) under varied laser illumination intensity from 0.02 to $39 \mathrm{~mW} \mathrm{~cm}^{-2}$, and $\mathbf{b}$ the corresponding transfer curves measured using Si back-gate under a constant collector to emitter bias $V_{\mathrm{CE}}=1 \mathrm{~V}$. c Responsivity and the extracted threshold voltage shift $\Delta V_{\text {th }}$ of the transistor at varied light illumination intensity. $\mathbf{d}$ Spatial photocurrent map of the npn bipolar phototransistor (Dev-3) under $V_{C E}=0$ and $1 \mathrm{~V}$. e compares the lateral distribution of photocurrent to the PFM phase pattern used to define the npn transistor. The reversed photocurrent polarity at $V_{C E}=0 \mathrm{~V}$ near emitter (E) and collector (C) terminals originates from the reverse $\mathrm{pn}$ junctions of emitter to base $(E-B)$ and base to collector $(B-C)$. Under $V_{C E}$ bias, most photocurrent is generated near the reversely biased base-collector (B-C) junction for the intense electric field there. $\mathbf{f}$ Comparison of the photodetection performance of MoS $_{2}$-based devices (filled symbols, this work and open symbols, literature: refs. 3,18,34,63-65), including photoconductors (square), photodiodes (diamond), bipolar phototransistors (circle), and other types of phototransistors (triangle)

ultimate device response speed depends on both material characteristics and device geometries. Further improved speed is likely attainable given higher carrier mobility in $\mathrm{MoS}_{2}$ and improved design on the width of base and collector, as they directly determine the overall carrier transit time in device. In Fig. 5b, the phototransistor behavior is further characterized by the transfer curves obtained by the back-gate field-effect modulation. As the current in the npn junction is limited by the generation rate of the minority electrons in the base, the asformed bipolar transistor manifests n-type conductance that increases with increasing $V_{\mathrm{g}}$ during field-effect measurements. When shining light on the device, the threshold voltage $V_{\text {th }}$ is apparently negatively shifted, implying an increase of electron concentration in the base by the forward injection from the emitter. The shift of threshold voltage is consolidated in another npn bipolar transistor defined on 8-nm-thick $\mathrm{MoS}_{2}$ device (Supplementary Fig. 12). The responsivity of the npn transistor is extracted and compared to the shift of $V_{\text {th }}$ under varied illumination intensity, as shown in Fig. $5 c$. $R$ closely follows the variation of $V_{\mathrm{th}}$, implying the important role of injected electrons in amplifying the photoresponse. $R$ slightly decreases with the negative shift of $V_{\text {th }}$ at higher light intensity, which is also usually found in other type phototransistors exhibiting a photogate effect. However, we emphasize that the origin of such dependence is different from the usual saturated charge trapping or separation in phototransistor, but due to the increasing recombination losses at the forward biased E-B junction under large injection ${ }^{28}$. In Fig. $5 \mathrm{~d}$, e, we present the photocurrent map for the present npn bipolar phototransistor under $V_{\mathrm{CE}}=0$ and $1 \mathrm{~V}$ to validate its operation principle. It is seen that photocurrent of reversed polarity appears near $\mathrm{C}$ and $\mathrm{E}$ terminal at $V_{\mathrm{CE}}=0 \mathrm{~V}$, while at $V_{\mathrm{CE}}=1 \mathrm{~V}$ the photocurrent is more efficiently generated near the reversely biased $\mathrm{B}-\mathrm{C}$ junction. This is consistent with the expected electric field strength in a device that eventually separates the photogenerated electron-hole pairs. The present photocurrent map also differs from other type of phototransistors that usually displays uniform photocurrent distribution within the photogate area ${ }^{60-62}$. Such difference in the two kinds of phototransistors is ascribed to their different gain generation mechanism, that is, via the lateral in-plane charge injection and the out-of-plane photovoltaic effects, respectively.

By virtue of the low dark current, the detector could be optimally operated without the assistant of external gate biases, yielding $R \sim 12 \mathrm{~A} \mathrm{~W}^{-1}$ at $0.2 \mathrm{~mW} \mathrm{~cm}^{-2}$ and high shot-noiselimited detectivity of $10^{13}$ Jones according to $D^{*}=R A^{-1 / 2}$ / $\left(2 e I_{\text {dark }}\right)^{-1 / 2}$, where $A$ is the device area. As a bipolar phototransistor, the device is found to exhibit 10 times higher responsivity over the initial photoconductor while preserving a fast response speed similar to the photodiode (see Fig. 5e). Compared to the photodiode, a substantial gain factor of 1000 is expected from the defined bipolar transistor to yield such an improvement to responsivity. The value is however substantially larger than any previous reported bipolar transistors based on buried gate ${ }^{28}$, lateral heterojunctions ${ }^{29}$, or vertically stacked heterojunctions ${ }^{34}$. As indicated in Fig. 5f, when compared to other $\mathrm{MoS}_{2}$ photodetectors in either photoconductors 3,18 , photo$\operatorname{diode}^{63}$, or the phototransistor configurations $\mathrm{s}^{34,64,65}$, the high gain value gives rise to competitive photodetection performance 
a
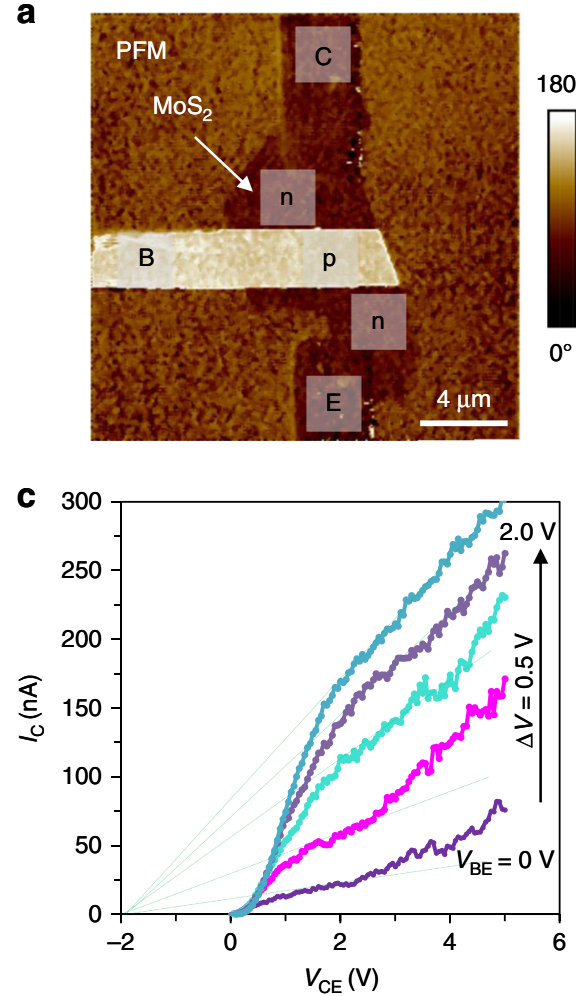

b

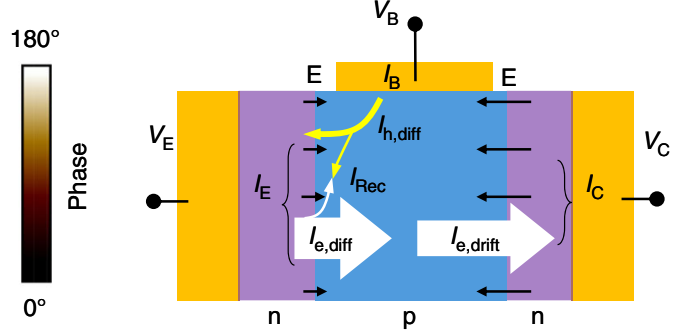

d

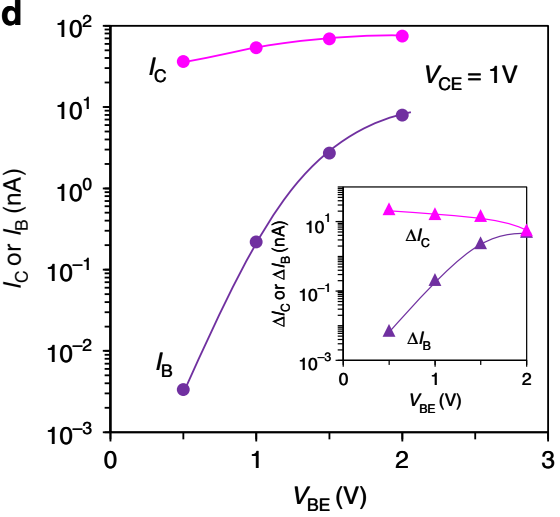

Fig. 6 Ferroelectric polarization-patterned three-terminal $\mathrm{MoS}_{2}$ npn bipolar transistor and its characteristic. a Piezoelectric force microscope (PFM) phase image of a three-terminal bipolar transistor with base connected to external terminal. $\mathbf{b}$ Illustration of the current components that contribute to the overall collector current $I_{C}$. The input base current $I_{\mathrm{B}}$ (contributed mainly by hole diffusion, $I_{\mathrm{h}}$, diff) is amplified by the triggered electron diffusion current at the emitter $\left(I_{\mathrm{e}}\right.$, diff $)$, which is then swept to the collector terminal $\left(I_{\mathrm{e}}\right.$, drift $)$ by the intense electric field (indicated by black arrows) at reversely biased junction. Recombination loss $\left(I_{\mathrm{Rec}}\right.$ ) during diffusion through base shall be suppressed to maximize the amplification ratio. c Output characteristic of the threeterminal bipolar transistor under varied base input voltages $V_{\mathrm{BE}}$. Increasing $V_{\mathrm{CE}}$ at a constant $V_{\mathrm{BE}}$ results in larger $I_{\mathrm{C}}$ due to the extended space charge region in reverse biased base-collector (B-C) junction and due to the reduction of effective base width, which promotes electron diffusion across the base. Extrapolation to $I_{C}=0$ under different $V_{\mathrm{BE}}$ yields a consistent early voltage of approximately $-2 \mathrm{~V}$. $\mathbf{d}$ Dependence of the collector $I_{\mathrm{C}}$ and base current $I_{\mathrm{B}}$ to $V_{\mathrm{BE}}$ input. The inset displays the extracted $\Delta \mathrm{I}_{\mathrm{C}}$ and $\Delta \mathrm{I}_{\mathrm{B}}$ at each $V_{\mathrm{BE}}$ step, from what a substantial gain $\left(\beta=\Delta \mathrm{I}_{\mathrm{C}} / \Delta \mathrm{I}_{\mathrm{B}}\right) \approx 3000$ is derived for the transistor action

by delivering simultaneously fast speed response speed and high responsivity (see detailed comparison in Supplementary Table 2).

In order to confirm the high performance of the bipolar transistor, we fabricate a three-terminal device by using $\mathrm{MoS}_{2}$ of similar thickness $\sim 5 \mathrm{~nm}$. Figure 6 a displays the PFM phase pattern of the FE polarization for the device. The base terminal is connected to metal electrode contact for a direct input of the base current $\left(I_{\mathrm{B}}\right)$, thus to probe the amplification at collector end. The current that contributed to the overall output at $V_{\mathrm{C}}>V_{\mathrm{B}}>V_{\mathrm{E}}$ is schematically illustrated in Fig. 6b. The output characteristic of the transistor is shown in Fig. 6c, which is measured by sweeping $V_{\mathrm{CE}}$ under different $V_{\mathrm{B}}$ input from 0 to $2 \mathrm{~V}$ and in the presence of the background laser of AFM feedback system. Here, the collector current $I_{\mathrm{C}}$ is significantly modulated by the input $V_{\mathrm{B}}$. Note that $I_{\mathrm{CE}}$ increased with increasing $V_{\mathrm{CE}}$ rather than being a constant due to the modulation to the base width by the extended space charge region at reverse biased $\mathrm{B}-\mathrm{C}$ junction ${ }^{59}$. Such base width modulation results in a small early voltage of $\sim 2 \mathrm{~V}$ (the extrapolated intersection with $V_{\mathrm{CE}}$ ), which is related to the low doping concentration in base. Slight increase of $V_{\mathrm{CE}}$ would dramatically modulate the effective base width and the diffusion current across the base. From the measured output characteristic, the amplification behavior of the bipolar transistor is extracted at $V_{\mathrm{CE}}=1 \mathrm{~V}$ and shown in Fig. $6 \mathrm{~d}$. The collector current $I_{\mathrm{C}}$ scaled with increasing $V_{\mathrm{BE}}$, and is 2-3 orders of magnitude larger than $I_{\mathrm{B}}$. The gain factor observed in photodetection could be probed from the magnitude of $I_{\mathrm{C}}$ variation while changing $I_{\mathrm{B}}$. As shown in the inset of Fig. $6 \mathrm{~d}$, when changing $V_{\mathrm{BE}}$ from 0 to $1 \mathrm{~V}$, we obtain an superior gain factor $\beta \sim 3000$, which is on the same order of magnitude as the expected amplification ratio in bipolar phototransistor as discussed above. The gain factor $\beta$ decreases gradually to 1 when $V_{\mathrm{BE}}$ is increased, following closely the case in photodetection that responsivity reduces at higher light illumination intensity. Interestingly, at $V_{\mathrm{B}}=1.5 \mathrm{~V}$, with both $\mathrm{B}-\mathrm{C}$ and $\mathrm{B}-\mathrm{E}$ junction forward biased, one still obtains $\beta \sim 10$. This clearly suggests the significant electron diffusion flux from emitter to collector in contrast to the hole flux in the same direction at the forward biased base to collector junction ${ }^{29}$. Our results therefore unambiguously confirm the high gain characteristic of FEcoupled bipolar phototransistor, which originates from the widely and fine-tuned doping states in $\mathrm{MoS}_{2}$.

\section{Conclusions}

In summary, we have demonstrated the facile reconfigurable customization of $\mathrm{MoS}_{2}$ optoelectronic devices using the rigid pn doping engineering enabled by switchable FE polarization. By polarizing the $\mathrm{MoS}_{2}$ device into homojunctions of pn diode and npn bipolar transistors, the device is configured into self-powered or high gain photodetectors of optimal performances without the assistance of external gate bias. The gate-free yet reconfigurable methodology introduced the great potential of exploiting locally 
coupled FE polarization in customizing high-performance optoelectronic devices based on the thriving 2D semiconductors and in the future their van der Waals heterojunctions, which in principle could offer even larger speed and gain product than present homojunctions. Further maturation of such strategy towards array-structured functional optoelectronic devices with high stability shall be viable based on predefined top-gate patterns or alternatively flexible electrical imprint methods.

\section{Methods}

Device fabrication. Few-layer $\mathrm{MoS}_{2}$ with thickness ranging from 2 to $5 \mathrm{~nm}$ are exfoliated from $\mathrm{MoS}_{2}$ single crystals (Nanjing MKNANO Tech. Co., Ltd.) using scotch tape and transferred to $\mathrm{SiO}_{2} / \mathrm{Si}$ substrate by polydimethylsiloxane stamp. The electrode contacts are made by $\mathrm{Cr} / \mathrm{Au}$ electrode. In the case of bottom electrode contacts, $\mathrm{Cr} / \mathrm{Au}(5 / 5 \mathrm{~nm})$ electrodes are first defined on the substrate using lithography processes with direct laser writing. The exfoliated $\mathrm{MoS}_{2}$ thin flakes are later transferred. The as-prepared devices are then annealed at $150{ }^{\circ} \mathrm{C}$ with $\mathrm{Ar}$ protection for $1 \mathrm{~h}$ to release stresses and improve electrical contact. The FE thin film of $\mathrm{P}(\mathrm{VDF}-\mathrm{TrFE})$ copolymer is then spin coated on the device using a solution of 2-5 wt\% P(VDF-TrFE) (Piezotech FC 25) dispersed in anhydrous N,N-dimethylformamide ( $99.8 \%$, Alfa Aesar). The copolymer film is then annealed at $135^{\circ} \mathrm{C}$ for $15 \mathrm{~min}$ to improve the crystallinity.

Local FE polarization. An AFM system (Dimension Icon, Bruker) is used to realize the FE poling using scanning conductive AFM tip with the $\mathrm{MoS}_{2}$ source and drain electrode biased by a poling voltage $V_{\mathrm{p}}$. A potential bias of $0.1 \mathrm{~V}$ is kept between source and drain for the in situ observation of the $\mathrm{MoS}_{2}$ conductance during the polarization scan. To obtain local polarization coupling to $\mathrm{MoS}_{2}, V_{\mathrm{p}}$ is programmed during the AFM scan.

Device characterization. The device after polarization is placed in a Lakeshore probe station (TTPX, Lakeshore) equipped with a semiconductor device analyzer (B1500A, Agilent) for electrical measurement. After AFM poling, the FE polarization in device tends to degrade within $1-2 \mathrm{~h}$ because of the lack of screening to depolarization field without top metal contacts. The device performance is therefore studied within this period; out of the period the device is repolarized at the same conditions for measurements. A 532-nm laser with tansistor-trasistor logic triggering is used as the light source for photodetection measurements, in which a power meter (FieldMaxII-TO, Coherent) is used to calibrate the light intensity. To probe the photoresponse speed, a $365 \mathrm{~nm}$ fast-switching LED (M365FP1, Thorlabs) and fast measurement unit (B1530, Agilent) is used.

\section{Data availability}

The data that support the plots within this paper and other findings of this study are available from the corresponding authors upon reasonable request.

Received: 16 October 2018 Accepted: 2 July 2019

Published online: 26 July 2019

\section{References}

1. Wang, Q. H., Kalantar-Zadeh, K., Kis, A., Coleman, J. N. \& Strano, M. S. Electronics and optoelectronics of two-dimensional transition metal dichalcogenides. Nat. Nanotechnol. 7, 699-712 (2012).

2. Radisavljevic, B., Radenovic, A., Brivio, J., Giacometti, V. \& Kis, A. Single-layer $\mathrm{MoS}_{2}$ transistors. Nat. Nanotechnol. 6, 147-150 (2011).

3. Lopez-Sanchez, O., Lembke, D., Kayci, M., Radenovic, A. \& Kis, A. Ultrasensitive photodetectors based on monolayer $\mathrm{MoS}_{2}$. Nat. Nanotechnol. 8, 497-501 (2013).

4. Geim, A. K. \& Grigorieva, I. V. Van der Waals heterostructures. Nature 499, 419-425 (2013).

5. Splendiani, A. et al. Emerging photoluminescence in monolayer $\mathrm{MoS}_{2}$. Nano Lett. 10, 1271-1275 (2010).

6. Radisavljevic, B. \& Kis, A. Mobility engineering and a metal-insulator transition in monolayer $\mathrm{MoS}_{2}$. Nat. Mater. 12, 815-820 (2013).

7. Cheng, R. et al. Few-layer molybdenum disulfide transistors and circuits for high-speed flexible electronics. Nat. Commun. 5, 5143 (2014).

8. Zhang, Y. J., Ye, J. T., Yomogida, Y., Takenobu, T. \& Iwasa, Y. Formation of a stable p-n junction in a liquid-gated $\mathrm{MoS}_{2}$ ambipolar transistor. Nano Lett. 13 , 3023-3028 (2013).

9. Costanzo, D., Jo, S., Berger, H. \& Morpurgo, A. F. Gate-induced superconductivity in atomically thin $\mathrm{MoS}_{2}$ crystals. Nat. Nanotechnol. 11, 339-344 (2016).

10. Chen, J. W. et al. A gate-free monolayer $\mathrm{WSe}_{2}$ pn diode. Nat. Commun. $\mathbf{9}$, 3143 (2018).
11. Lee, H. S. et al. $\mathrm{MoS}_{2}$ nanosheets for top-gate nonvolatile memory transistor channel. Small 8, 3111-3115 (2012).

12. Wang, X. et al. Ultrasensitive and broadband $\mathrm{MoS}_{2}$ photodetector driven by ferroelectrics. Adv. Mater. 27, 6575-6581 (2015).

13. Mak, K. F. et al. Tightly bound trions in monolayer $\mathrm{MoS}_{2}$. Nat. Mater. 12, 207-211 (2013).

14. Kim, S. et al. High-mobility and low-power thin-film transistors based on multilayer $\mathrm{MoS}_{2}$ crystals. Nat. Commun. 3, 1011 (2012).

15. Das, S., Chen, H. Y., Penumatcha, A. V. \& Appenzeller, J. High performance multilayer $\mathrm{MoS}_{2}$ transistors with scandium contacts. Nano Lett. 13, 100-105 (2013).

16. Lipatov, A., Sharma, P., Gruverman, A. \& Sinitskii, A. Optoelectrical molybdenum disulfide $\left(\mathrm{MoS}_{2}\right)$-ferroelectric memories. ACS Nano $\mathbf{9}$, 8089-8098 (2015).

17. Sundaram, R. S. et al. Electroluminescence in single layer $\mathrm{MoS}_{2}$. Nano Lett. 13, 1416-1421 (2013)

18. Kufer, D. \& Konstantatos, G. Highly sensitive, encapsulated $\mathrm{MoS}_{2}$ photodetector with gate controllable gain and speed. Nano Lett. 15, 7307-7313 (2015).

19. Huang, Y. et al. Van der Waals coupled organic molecules with monolayer $\mathrm{MoS}_{2}$ for fast response photodetectors with gate-tunable responsivity. ACS Nano 12, 4062-4073 (2018).

20. Jin, H. J., Yoon, W. Y. \& Jo, W. Control of work function of $\mathrm{MoS}_{2}$ with ferroelectric polarization in honeycomb-like heterostructure. Appl. Phys. Lett. 110, 191607 (2017).

21. Lipatov, A. et al. Polarization-dependent electronic transport in graphene/Pb $(\mathrm{Zr}, \mathrm{Ti}) \mathrm{O}_{3}$ ferroelectric field-effect transistors. Adv. Electron. Mater. 3, 1700020 (2017).

22. Xiao, Z., Song, J., Ferry, D. K., Ducharme, S. \& Hong, X. Ferroelectricdomain-patterning-controlled Schottky junction state in monolayer $\mathrm{MoS}_{2}$. Phys. Rev. Lett. 118, 236801 (2017)

23. Zhou, C. J. \& Chai, Y. Ferroelectric-gated two-dimensional-material-based electron devices. Adv. Electron. Mater. 3, 1600400 (2017).

24. Zhang, X. W. et al. $\mathrm{MoS}_{2}$ field-effect transistors with lead zirconate-titanate ferroelectric gating. IEEE Electron Dev. Lett. 36, 784-786 (2015)

25. Nguyen, A. et al. Toward ferroelectric control of monolayer $\mathrm{MoS}_{2}$. Nano Lett. 15, 3364-3369 (2015)

26. Luo, P. et al. Doping engineering and functionalization of two-dimensional metal chalcogenides. Nanoscale Horiz. 4, 26-51 (2019).

27. Pham, V. P. \& Yeom, G. Y. Recent advances in doping of molybdenum disulfide: industrial applications and future prospects. Adv. Mater. 28, 9024-9059 (2016).

28. Agnihotri, P., Dhakras, P. \& Lee, J. U. Bipolar junction transistors in twodimensional $\mathrm{WSe}_{2}$ with large current and photocurrent gains. Nano Lett. 16, 4355-4360 (2016)

29. Lin, C. Y. et al. Atomic-monolayer two-dimensional lateral quasiheterojunction bipolar transistors with resonant tunneling phenomenon. ACS Nano 11, 11015-11023 (2017).

30. Yang, T. et al. Van der Waals epitaxial growth and optoelectronics of largescale $\mathrm{WSe}_{2} / \mathrm{SnS}_{2}$ vertical bilayer p-n junctions. Nat. Commun. 8, 1906 (2017).

31. Mahjouri-Samani, M. et al. Patterned arrays of lateral heterojunctions within monolayer two-dimensional semiconductors. Nat. Commun. 6, 7749 (2015).

32. Huang, M. et al. Multifunctional high-performance van der Waals heterostructures. Nat. Nanotechnol. 12, 1148-1154 (2017).

33. Zhang, C. et al. Strain distributions and their influence on electronic structures of $\mathrm{WSe}_{2}-\mathrm{MoS}_{2}$ laterally strained heterojunctions. Nat. Nanotechnol. 13, 152-158 (2018)

34. Ye, L., Li, H., Chen, Z. \& Xu, J. Near-infrared photodetector based on $\mathrm{MoS}_{2} /$ black phosphorus heterojunction. ACS Photonics 3, 692-699 (2016).

35. Kobayashi, T., Hori, N., Nakajima, T. \& Kawae, T. Electrical characteristics of $\mathrm{MoS}_{2}$ field-effect transistor with ferroelectric vinylidene fluoridetrifluoroethylene copolymer gate structure. Appl. Phys. Lett. 108, 132903 (2016).

36. Nourbakhsh, A., Zubair, A., Joglekar, S., Dresselhaus, M. \& Palacios, T. Subthreshold swing improvement in $\mathrm{MoS}_{2}$ transistors by the negativecapacitance effect in a ferroelectric Al-doped- $\mathrm{HfO}_{2} / \mathrm{HfO}_{2}$ gate dielectric stack. Nanoscale 9, 6122-6127 (2017).

37. Wang, X. D. et al. Ferroelectric FET for nonvolatile memory application with two-dimensional $\mathrm{MoSe}_{2}$ channels. 2D Mater. 4, 025036 (2017).

38. Yin, L. et al. Ferroelectric-induced carrier modulation for ambipolar transition metal dichalcogenide transistors. Appl. Phys. Lett. 110, 123106 (2017).

39. Gregorio, R. \& Botta, M. M. Effect of crystallization temperature on the phase transitions of $\mathrm{P}(\mathrm{VDF} / \mathrm{TrFE})$ copolymers. J. Polym. Sci. B 36, 403-414 (1998).

40. Ducharme, S. et al. Intrinsic ferroelectric coercive field. Phys. Rev. Lett. 84, 175-178 (2000).

41. Chen, Y. et al. Optoelectronic properties of few-layer $\mathrm{MoS}_{2}$ FET gated by ferroelectric relaxor polymer. ACS Appl. Mater. Interfaces 8, 32083-32088 (2016). 
42. Kim, C. et al. Fermi level pinning at electrical metal contacts of monolayer molybdenum dichalcogenides. ACS Nano 11, 1588-1596 (2017).

43. Liu, X. et al. P-type polar transition of chemically doped multilayer $\mathrm{MoS}_{2}$ transistor. Adv. Mater. 28, 2345-2351 (2016).

44. Rajapitamahuni, A., Hoffman, J., Ahn, C. H. \& Hong, X. Examining graphene field effect sensors for ferroelectric thin film studies. Nano Lett. 13, 4374-4379 (2013).

45. Dolui, K., Rungger, I., Das Pemmaraju, C. \& Sanvito, S. Possible doping strategies for $\mathrm{MoS}_{2}$ monolayers: an ab initio study. Phys. Rev. B 88, 075420 (2013).

46. Qiu, H. et al. Hopping transport through defect-induced localized states in molybdenum disulphide. Nat. Commun. 4, 2642 (2013).

47. Ghatak, S. \& Ghosh, A. Observation of trap-assisted space charge limited conductivity in short channel $\mathrm{MoS}_{2}$ transistor. Appl. Phys. Lett. 103, 122103 (2013).

48. Wang, F. et al. Tunable GaTe- $\mathrm{MoS}_{2}$ van der Waals p-n junctions with novel optoelectronic performance. Nano Lett. 15, 7558-7566 (2015).

49. Choi, M. S. et al. Lateral $\mathrm{MoS}_{2}$ p-n junction formed by chemical doping for use in high-performance optoelectronics. ACS Nano 8, 9332-9340 (2014).

50. Sutar, S. et al. Reconfigurable p-n junction diodes and the photovoltaic effect in exfoliated $\mathrm{MoS}_{2}$ films. Appl. Phys. Lett. 104, 122110 (2014).

51. Castellanos-Gomez, A., Quereda, J., van der Meulen, H. P., Agrait, N. \& RubioBollinger, G. Spatially resolved optical absorption spectroscopy of single- and few-layer $\mathrm{MoS}_{2}$ by hyperspectral imaging. Nanotechnology 27, 115705 (2016).

52. Svatek, S. A. et al. Gate tunable photovoltaic effect in $\mathrm{MoS}_{2}$ vertical $\mathrm{p}-\mathrm{n}$ homostructures. J. Mater. Chem. C 5, 854-861 (2017).

53. Li, M. Y. et al. Epitaxial growth of a monolayer $\mathrm{WSe}_{2}-\mathrm{MoS}_{2}$ lateral p-n junction with an atomically sharp interface. Science 349, 524-528 (2015).

54. Furchi, M. M., Polyushkin, D. K., Pospischil, A. \& Mueller, T. Mechanisms of photoconductivity in atomically thin $\mathrm{MoS}_{2}$. Nano Lett. 14, 6165-6170 (2014).

55. Wang, $\mathrm{G}$. et al. Seamless lateral graphene $\mathrm{p}-\mathrm{n}$ junctions formed by selective in situ doping for high-performance photodetectors. Nat. Commun. 9, 5168 (2018).

56. Mueller, T., Xia, F., Freitag, M., Tsang, J. \& Avouris, P. Role of contacts in graphene transistors: a scanning photocurrent study. Phys. Rev. B 79, 245430 (2009).

57. Yi, Y. et al. A study of lateral Schottky contacts in $\mathrm{WSe}_{2}$ and $\mathrm{MoS}_{2}$ field effect transistors using scanning photocurrent microscopy. Nanoscale 7 , 15711-15718 (2015)

58. Long, M., Wang, P., Fang, H. \& Hu, W. Progress, challenges, and opportunities for 2D material based photodetectors. Adv. Funct. Mater. https://doi.org/10.1002/adfm.201803807 (2018).

59. Neamen, D. A. Semiconductor Physics and Devices: Basic Principles 4th edn (McGraw-Hill Education, New York, 2017).

60. Ye, L. et al. Highly polarization sensitive infrared photodetector based on black phosphorus-on- $\mathrm{WSe}_{2}$ photogate vertical heterostructure. Nano Energy 37, 53-60 (2017).

61. Liu, F. \& Kar, S. Quantum carrier reinvestment-induced ultrahigh and broadband photocurrent responses in graphene-silicon junctions. ACS Nano 8, 10270-10279 (2014)

62. Thakar, K. et al. Multilayer $\mathrm{ReS}_{2}$ photodetectors with gate tunability for high responsivity and high-speed applications. ACS Appl. Mater. Interfaces 10, 36512-36522 (2018).

63. Zhang, X. et al. Poly(4-styrenesulfonate)-induced sulfur vacancy self-healing strategy for monolayer $\mathrm{MoS}_{2}$ homojunction photodiode. Nat. Commun. 8, 15881 (2017).
64. Tsai, D. S. et al. Few-layer $\mathrm{MoS}_{2}$ with high broadband photogain and fast optical switching for use in harsh environments. ACS Nano 7, 3905-3911 (2013).

65. Huo, N. \& Konstantatos, G. Ultrasensitive all-2D $\mathrm{MoS}_{2}$ phototransistors enabled by an out-of-plane $\mathrm{MoS}_{2} \mathrm{PN}$ homojunction. Nat. Commun. 8, 572 (2017).

\section{Acknowledgements}

This work was supported by National Natural Science Foundation of China (Grant Nos 61804059, 21825103, and 51727809), National Key Research and Development Program of "Strategic Advanced Electronic Materials" (Grant No. 2016YFB0401100), and the Fundamental Research Funds for the Central University (Grant No. 2019kfyXMBZ018). F.W.Z. owes special thanks to Dr. M.M. Yang at the University of Warwick for the discussion.

\section{Author contributions}

F.W.Z. and T.Y.Z. conceived the idea. L.L., F.J.X., X.J.X., and N.Z. prepared and characterized $\mathrm{P}(\mathrm{VDF}-\mathrm{TrFE})$ polymers, $\mathrm{MoS}_{2}$, WSe $\mathrm{W}_{2}$ flakes, and the devices. L.L., Q.F.Z., and Y.H. performed PFM polarization, device characterization, and photocurrent mapping. F. W.Z., T.Y.Z., and L.L. analyzed the data and wrote the manuscript together with discussion with all authors.

\section{Additional information}

Supplementary Information accompanies this paper at https://doi.org/10.1038/s41467019-11328-0.

Competing interests: The authors declare no competing interests.

Reprints and permission information is available online at http://npg.nature.com/ reprintsandpermissions/

Peer review information: Nature Communications thanks Chung-Lin Wu and other anonymous reviewers for their contribution to the peer review of this work. Peer reviewer reports are available.

Publisher's note: Springer Nature remains neutral with regard to jurisdictional claims in published maps and institutional affiliations.

Open Access This article is licensed under a Creative Commons Attribution 4.0 International License, which permits use, sharing, adaptation, distribution and reproduction in any medium or format, as long as you give appropriate credit to the original author(s) and the source, provide a link to the Creative Commons license, and indicate if changes were made. The images or other third party material in this article are included in the article's Creative Commons license, unless indicated otherwise in a credit line to the material. If material is not included in the article's Creative Commons license and your intended use is not permitted by statutory regulation or exceeds the permitted use, you will need to obtain permission directly from the copyright holder. To view a copy of this license, visit http://creativecommons.org/ licenses/by/4.0/

(C) The Author(s) 2019 